\title{
Anisotropic scattering kernel : generalized and modified Maxwell boundary conditions
}

\author{
S. Kokou Dadzie* and J. Gilbert Méolans ${ }^{\dagger}$ \\ Polytech'Marseille, \\ Département de Mécanique Energétique - UMR CNRS 6595 \\ 5, rue Enrico Fermi 13453 Marseille cedex 13 France
}

(Dated: 28 October 2004; accepted for publication 03 February 2004)

This paper presents a model of a scattering kernel of boundary conditions for the Boltzmann equation. The proposed scattering kernel is based on an anisotropic accommodation argument. Three parameters equal to the momentum accommodation coefficients are shown as characterizing the influence of each direction. First the new scattering kernel is derived from a phenomenological criticism of the first form of the scattering kernel proposed by Maxwell; then the same result is established from an analytic approach based on the spectral nature of the linear integral operator associated to the scattering kernel problem. As a result, the model provides a correct form of scattering kernel to handle the influence of each direction in particle collisions with the wall. Finally independent accommodation of each internal mode is added to extend the model to the case of polyatomic gases.

PACS numbers: 47.45.-n,51.10.+y

Keywords: kinetic theory; gas-wall interaction; scattering kernel; accommodation coefficients; Boltzmann equation

\footnotetext{
*Electronic address: Kokou.Dadzie@polytech.univ-mrs.fr

${ }^{\dagger}$ Electronic address: Gilbert.Meolans@polytech.univ-mrs.fr; URL: http://www.polytech.univ-mrs . fr
} 


\section{INTRODUCTION}

The problem of writing good boundary conditions for the Boltzmann equation in rarefied gas flows, is to find an operator called scattering kernel in kinetic theory. The first known scattering kernel was proposed by Maxwell and is based on phenomenological argument [1]. However, in various situations this kernel fails to reproduce correctly the phenomena occurring at the wall $[2,3]$. Indeed the Maxwell boundary condition corresponds to an isotropic conception of the reflection at the wall; so, in this approach, the three velocity components are considered as equivalent in the accommodation process.

Another class of scattering kernel is the CL (Cercignani-Lampis) model by Cercignani et al. $[3,4]$. The authors extend the research to a more general field of operators and obtain a more flexible model. However as is well-known this class of scattering kernels are not totally efficient to describe physically gas behavior close to the wall [4]. Moreover, some results in particle simulation of rarefied gas flows seem to show that none of the existing models of scattering kernel can reproduce all the features of the real gas dynamics [5].

Generally the existing models of scattering kernel do not describe the interplay between the different degrees of freedom (i.e. the three velocity components of the particle and its internal energy modes) in interaction with the wall [5]. This would explain the partial inefficiency of these models to reproduce the behavior of high speed non-equilibrium flows near the wall. The main purpose of this paper is to propose a new model of scattering kernel by considering more possible reflection types of particles at the wall. In this way we attempt to eliminate any isotropic character of momentum accommodation in the velocity reflection process, and we introduce three coefficients to take into account the influence of the velocity directions.

From section II to section VI, we consider only unstructured atom-like molecules. In section II the basic conditions required for a scattering kernel are briefly analyzed and discussed. In section III the Maxwell phenomenological argument is recalled and we present the new model. In section IV we develop a consistent analytical approach to build the new 
scattering kernel. In section $\mathbf{V}$ the coefficients introduced in the modelling are shown to equal the well known momentum accommodation coefficients. Finally in section VI the new kernel is involved in a more complete model characterizing molecules with internal modes.

\section{CONDITIONS REQUIRED FOR THE SCATTERING KERNELS}

Let us consider a particle hitting the wall, as shown in FIG.1. $V^{\prime}$ is the velocity of the impinging gas particle referred to the wall, $V^{\prime}=\left(V_{x}^{\prime}, V_{y}^{\prime}, V_{z}^{\prime}\right) \in\left\{\Omega^{\prime}=\mathbb{R}_{-} \times \mathbb{R} \times \mathbb{R}\right\}$ and $V$ the velocity of the reflected one referred to the wall, $V=\left(V_{x}, V_{y}, V_{z}\right) \in\left\{\Omega=\mathbb{R}_{+} \times \mathbb{R} \times \mathbb{R}\right\}$. These velocities reduce to peculiar velocities when the slip velocity at the wall is neglected. $V_{R}$ is defined as $V_{R}=\left(-V_{x}, V_{y}, V_{z}\right)$.

The kernel, $B\left(V^{\prime}, V\right)$, is the density of probability that a molecule impinging the wall at any point $X$ of the wall with velocity $V^{\prime}$ is reflected at the same point with velocity $V$. This kernel, which turns the impinging particles at the wall into reflected ones, must satisfy some basic physical conditions. The least obvious of them is the reciprocity relation. This relation is a necessary condition for a good scattering kernel in the kinetic theory of gases $[6,7]$.

According to the scattering kernel probability density property, kernel $B\left(V^{\prime}, V\right)$ must satisfy:

$$
B\left(V^{\prime}, V\right) \geq 0
$$

and also the normalization condition

$$
\int_{\Omega} B\left(V^{\prime}, V\right) d_{V}=1
$$

Finally the balance of particles hitting the wall at position $X$ and reflected with velocity $V$ at a given time may be written [8]:

$$
V_{x} f(V)=\int_{\Omega^{\prime}}\left|V_{x}^{\prime}\right| f\left(V^{\prime}\right) B\left(V^{\prime}, V\right) d_{V^{\prime}}
$$

where $f(V)$ is the gas particle distribution function given by the Boltzmann equation. 
In the thermodynamic equilibrium state between the gas and the wall at the same temperature, the time reversibility assumption of the thermodynamic equilibrium state may be formulated as follows $[3,7,9]$ : the number of gas particles which hit the wall during time $t$, with velocity $V^{\prime}$ and reflect with velocity $V$ at position $X$ of the wall, is equal to the number of particles with incident velocity $-V$ reflected with velocity $-V^{\prime}$ at the same time $t$, at the same position $X$ of the wall. This may be written:

$$
\left|V_{x}^{\prime}\right| f_{0}\left(V^{\prime}\right) B\left(V^{\prime}, V\right)=\left|V_{x}\right| f_{0}(-V) B\left(-V,-V^{\prime}\right)
$$

where $f_{0}(V)=\frac{n}{\left(C_{w} \sqrt{\pi}\right)^{3}} e^{-\frac{\|V\|^{2}}{C_{w}^{2}}}$ is the Maxwellian distribution function of the gas in equilibrium state at the temperature of the wall. $C_{w}^{2}=\frac{2 k T_{w}}{m}$, where $T_{w}$ is the wall temperature, $k$ is the Boltzmann constant, $m$ the molecular mass of gaseous particles and $n$ their numerical density. The time reversibility assumption means that all detailed balancing of energy exchange between the gas and the wall in the thermodynamic equilibrium state at any time $t$ is equal to zero. This state of thermodynamic equilibrium between the gas and the wall at the same temperature is a reference state. Owing to this fact, any scattering kernel in kinetic theory must be validated by the property (4), called Reciprocity Relation.

Note that the normalization condition (2) is equivalent to:

$$
\int_{\Omega^{\prime}} B\left(-V,-V_{R}^{\prime}\right) d_{V^{\prime}}=1
$$

From this remark, the reciprocity relation also leads to:

$$
\int_{\Omega^{\prime}}\left|V_{x}^{\prime}\right| f_{0}\left(V^{\prime}\right) B\left(V_{R}^{\prime}, V\right) d_{V^{\prime}}=\left|V_{x}\right| f_{0}(-V)
$$

It is also necessary to note that the required conditions formulated above characterize a physical situation at the wall involving the conservation of the particle flux and then a binary and short interaction between the solid atoms and gaseous particles [9]. So the model presented here excludes the gas dissociation and various steps involved in catalysis (chemical reaction at the wall, adsorption etc...). 


\section{PHENOMENOLOGICAL APPROACH TO SCATTERING KERNELS}

\section{A. Maxwell scattering kernel}

The first form of scattering kernel proposed by Maxwell is given by:

$$
B_{M}\left(V^{\prime}, V\right)=(1-\alpha) \delta\left(V-V_{R}^{\prime}\right)+\alpha \frac{2}{C_{w}^{4} \pi} V_{x} e^{-\frac{\|V\|^{2}}{C_{w}^{2}}} .
$$

$B_{M}\left(V^{\prime}, V\right)$ is a linear combination of two elementary kernels: the dirac function for the specular reflection part and the exponential function for the diffuse reflection part at temperature $T_{w}$ of the wall; $\alpha$, called accommodation coefficient, represents the weight of the diffusion in the gas collision process at the wall.

Before writing this scattering kernel Maxwell made several phenomenological comments on the nature of the surface in contact with the gas, which can be found in the appendix of reference [1]. Firstly he assumed the surface to be a "perfectly elastic smooth surface", then each molecule striking the surface had its normal component reversed while the other components were not altered by the impact; the scattering kernel in this case was simply given by $\delta\left(V-V_{R}^{\prime}\right)$. Secondly he assumed the surface to be a perfectly absorbing surface so that each particle hitting the wall was absorbed before being re-emitted; and the velocity of each re-emitted particle was oriented from the surface towards the gas. But the probability of any particular magnitude and direction of its velocity would be the same as in a gas at rest in the thermal equilibrium state at the temperature of the wall; in this case, this led to the diffusive scattering kernel given by $\frac{2}{C_{w}^{4} \pi} V_{x} e^{-\frac{\|V\|^{2}}{C_{w}^{2}}}$.

Finally Maxwell decided to consider the real reflecting surfaces as an intermediate surface between these two extreme perfect surfaces and then proposed the linear combination (5).

In our view, the scattering process on a real surface must be more complicated than those described by the sum of the scattering kernels of the two extreme perfect surfaces. It is easily seen that the two elementary scattering kernels written for the two perfect surfaces are isotropic ones; this is reasonable for these two types of perfect surfaces. Then using a linear combination of these two elementary scattering kernels, the result will still be an 
isotropic one; but we cannot be sure that a scattering kernel characterizing any real surface is an isotropic one. Moreover, in the kernel (5), the weight of the diffusion in any direction is represented by the same coefficient $\alpha$ : this description cannot be considered as correct, as pointed out by various authors $[2,10]$.

\section{B. Our scattering kernel proposal}

In order to write a general scattering kernel for any kind of real surface, we return to the investigation of various possible types of accommodation which can occur during a particle collision with the wall. Then we have to consider a more general type of accommodation than the isotropic one, in which of course the normal component of the incoming particle velocity must be reversed during the collision. We can thus assume elementary processes where diffusive and specular reflection are mixed: each of the velocity components can be altered or not by the wall independently of others. This argument leads us to consider the eight elementary operators listed below:

$$
\begin{gathered}
B_{0}\left(V^{\prime}, V\right)=\delta\left(V_{x}+V_{x}^{\prime}\right) \delta\left(V_{y}-V_{y}^{\prime}\right) \delta\left(V_{z}-V_{z}^{\prime}\right), \\
B_{y z}\left(V^{\prime}, V\right)=\frac{1}{\pi C_{w}^{2}} \delta\left(V_{x}+V_{x}^{\prime}\right) e^{-\frac{V_{y}^{2}}{C_{w}^{2}}} e^{-\frac{V_{z}^{2}}{C_{w}^{2}}}, \\
B_{x z}\left(V^{\prime}, V\right)=\frac{2}{C_{w}^{3} \sqrt{\pi}} V_{x} \delta\left(V_{y}-V_{y}^{\prime}\right) e^{-\frac{V_{x}^{2}}{C_{w}^{2}}} e^{-\frac{V_{z}^{2}}{C_{w}^{2}}}, \\
B_{x y}\left(V^{\prime}, V\right)=\frac{2}{C_{w}^{3} \sqrt{\pi}} V_{x} \delta\left(V_{z}-V_{z}^{\prime}\right) e^{-\frac{V_{x}^{2}}{C_{w}^{2}}} e^{-\frac{V_{y}^{2}}{C_{w}^{2}}} \\
B_{x y z}\left(V^{\prime}, V\right)=\frac{2}{\pi C_{w}^{4}} V_{x} e^{-\frac{V_{x}^{2}}{C_{w}^{2}}} e^{-\frac{V_{y}^{2}}{C_{w}^{2}}} e^{-\frac{V_{z}^{2}}{C_{w}^{2}}} \\
B_{z}\left(V^{\prime}, V\right)=\frac{1}{C_{w} \sqrt{\pi}} \delta\left(V_{x}+V_{x}^{\prime}\right) \delta\left(V_{y}-V_{y}^{\prime}\right) e^{-\frac{V_{z}^{2}}{C_{w}^{2}}}
\end{gathered}
$$




$$
\begin{aligned}
& B_{y}\left(V^{\prime}, V\right)=\frac{1}{C_{w} \sqrt{\pi}} \delta\left(V_{x}+V_{x}^{\prime}\right) \delta\left(V_{z}-V_{z}^{\prime}\right) e^{-\frac{V_{y}^{2}}{C_{w}^{2}}}, \\
& \mathrm{~B}_{x}\left(V^{\prime}, V\right)=\frac{2}{C_{w}^{2}} V_{x} \delta\left(V_{y}-V_{y}^{\prime}\right) \delta\left(V_{z}-V_{z}^{\prime}\right) e^{-\frac{V_{x}^{2}}{C_{w}^{2}}} .
\end{aligned}
$$

It is easy to show that each of these elementary kernels satisfies positivity and normalization conditions. These kernels also satisfy the reciprocity relation (more details are given in appendix A). The coefficients appearing in front of each operator are normalizing coefficients obtained by calculating $\int_{\Omega} B_{\kappa}\left(V^{\prime}, V\right) d_{V}$.

Each of these elementary kernels represents a particular possible situation of accommodation. Then the complete scattering kernel will be a linear combination of the elementary kernels in which the combination coefficients will represent the weight of each kind of accommodation at the wall. This complete scattering kernel may be written:

$$
B\left(V^{\prime}, V\right)=\sum_{\kappa} \mu_{\kappa} B_{\kappa}\left(V^{\prime}, V\right)
$$

with

$$
\sum_{\kappa} \mu_{\kappa}=1
$$

If we introduce three coefficients, $\alpha_{j}(j=1,2,3)$ satisfying $\alpha_{j} \in[0,1]$, to quantify the influence of each direction in a particle accommodation process by the wall, and taking into account condition (8), the coefficients $\mu_{\kappa}$ may be written:

$$
\begin{array}{r}
\mu_{x z}=\alpha_{x} \alpha_{z}\left(1-\alpha_{y}\right), \quad \mu_{x y}=\alpha_{x} \alpha_{y}\left(1-\alpha_{z}\right), \quad \mu_{y z}=\alpha_{y} \alpha_{z}\left(1-\alpha_{x}\right) \\
\mu_{x}=\alpha_{x}\left(1-\alpha_{y}\right)\left(1-\alpha_{z}\right), \quad \mu_{x y z}=\alpha_{x} \alpha_{y} \alpha_{z}, \quad \mu_{0}=\left(1-\alpha_{x}\right)\left(1-\alpha_{y}\right)\left(1-\alpha_{z}\right) \\
\mu_{y}=\alpha_{y}\left(1-\alpha_{x}\right)\left(1-\alpha_{z}\right), \quad \mu_{z}=\alpha_{z}\left(1-\alpha_{x}\right)\left(1-\alpha_{y}\right) .
\end{array}
$$

According to this last relationship, the complete scattering kernel (7) satisfies the positivity and the normalization properties. It obviously satisfies the reciprocity relation since the elementary kernels satisfy it. 
It is clear that neglecting the elementary kernels with incomplete diffusion or incomplete specular reflection in the full scattering kernel (7), the result reduces to the sum of the kernels (6a) and (6b) which represents the Maxwell scattering kernel. Moreover, it is easy to show that the Maxwell boundary conditions give satisfactory results for values of $\alpha$ close to 1: indeed, if the gas is in a state very close to thermodynamic equilibrium at the temperature of the wall, the most important reflection is the reflection with complete accommodation in all directions.

In the following section, an analytical method to build the complete scattering kernel (7) is presented.

\section{ANALYTICAL FORMULATION OF THE NEW SCATTERING KERNEL}

Let us consider the problem of finding operator $B\left(V^{\prime}, V\right)$, satisfying the conditions listed in section II, and let us write the transformation

$$
K\left(V, V^{\prime}\right)=\left[\left|V_{x}^{\prime}\right| f_{0}\left(V^{\prime}\right)\right]^{\frac{1}{2}}\left[\left|V_{x}\right| f_{0}(V)\right]^{\frac{-1}{2}} B\left(V_{R}^{\prime}, V\right)
$$

Since $f_{0}(V)$ is a known function the problem of finding $B\left(V^{\prime}, V\right)$ is equivalent to finding $K\left(V, V^{\prime}\right)$. Instead of studying the problem in $K\left(V, V^{\prime}\right)$, we can study the linear integral associated operator $A$ defined by the relation:

$$
A(\psi)=\int_{\Omega^{\prime}} K\left(V, V^{\prime}\right) \psi\left(V^{\prime}\right) d_{V^{\prime}}
$$

This integral operator is defined in the Hilbert space of square summable functions of $V$ noted $L^{2}(\Omega)$ (i.e. $\psi(V) \in L^{2}(\Omega)$ ), where the scalar product is defined as follows:

$$
<\psi, \varphi>=\int_{\Omega} \psi(V) \varphi(V) d_{V} .
$$

Therefore the problem is reduced to an eigenvalue problem, and then to discussing the spectral nature of operator $A$.

It is to be noted that the above formulation of the scattering kernel problem, through the transformation (10) is especially convenient to solve the Linearized form of the Boltzman Equation (LBE) [11, 12]. 
Assuming that the operator $A$ has a purely discrete spectrum, its kernel $K\left(V, V^{\prime}\right)$ can be written:

$$
K\left(V, V^{\prime}\right)=\sum_{n=0}^{\infty} \lambda^{n} \psi^{n}(V) \psi^{n}\left(V^{\prime}\right),
$$

where $\psi^{n}(V) \in L^{2}(\Omega)$ is an eigenfunction of the operator $A$ and $\lambda_{n}$ its corresponding eigenvalue. According to positivity and normalization conditions required in $B\left(V^{\prime}, V\right)$, the operator $A$ must be non-negative and its eigenvalues satisfy $\lambda^{n} \in[0,1]$ for any $n \in \mathbb{N}$.

From now the eigenfunction $\psi^{n}(V)$ is assumed to be in the form:

$$
\psi_{r}\left(V_{x}\right) \psi_{l}\left(V_{y}\right) \psi_{m}\left(V_{z}\right)=\prod_{j=1}^{3} \psi_{k_{j}}\left(V_{j}\right)
$$

Since the eigenfunction $\psi^{n}(V)$ is a square summable function, we have $\psi_{k_{j}}\left(V_{j}\right) \in L^{2}\left(\Omega_{j}\right)$, where $\Omega_{j}$ denotes the scalar space associated to $V_{j}$. Moreover, the family of the function set $\psi_{k_{j}}\left(V_{j}\right), k_{j} \in \mathbb{N}$ must be a function basis of the Hilbert space $L^{2}\left(\Omega_{j}\right)$ (note that this assumption will lead immediately to the coming property (16)). The eigenvalues of operator $A$ become $\lambda_{r l m}$, and we can assume $\lambda_{r l m}=\lambda_{r} \lambda_{l} \lambda_{m}$, in respect to the respective scalar products in the different Hilbert spaces $L^{2}\left(\Omega_{j}\right)$ and $L^{2}(\Omega)$. Finally the expression (12) can be replaced by:

$$
K\left(V, V^{\prime}\right)=\sum_{r, l, m} \lambda_{r} \lambda_{l} \lambda_{m} \psi_{r}\left(V_{x}\right) \psi_{l}\left(V_{y}\right) \psi_{m}\left(V_{z}\right) \psi_{r}\left(V_{x}^{\prime}\right) \psi_{l}\left(V_{y}^{\prime}\right) \psi_{m}\left(V_{z}^{\prime}\right)
$$

The sum in the right member of (13) can be written as the product of three infinite sums:

$$
K\left(V, V^{\prime}\right)=\prod_{j=1}^{3} \sum_{k=0}^{\infty} \lambda_{k_{j}} \psi_{k_{j}}\left(V_{j}\right) \psi_{k_{j}}\left(V_{j}^{\prime}\right) .
$$

Let us put

$$
\begin{aligned}
& \psi_{0_{x}}\left(V_{x}\right)=\frac{\sqrt{2}}{C_{w}}\left|V_{x}\right|^{\frac{1}{2}} e^{\frac{-V_{x}^{2}}{2 C_{w}^{2}}}, \\
& \psi_{0_{y}}\left(V_{y}\right)=\left(C_{w} \sqrt{\pi}\right)^{\frac{-1}{2}} e^{\frac{-V_{y}^{2}}{2 C_{w}^{2}}}, \\
& \psi_{0_{z}}\left(V_{z}\right)=\left(C_{w} \sqrt{\pi}\right)^{\frac{-1}{2}} e^{\frac{-V_{z}^{2}}{2 C_{w}^{2}}} .
\end{aligned}
$$

Each of $\psi_{0_{j}}\left(V_{j}\right)$ functions satisfies:

$$
\left\|\psi_{0_{j}}\left(V_{j}\right)\right\|_{j}^{2}=\int_{\Omega_{j}}\left[\psi_{0_{j}}\left(V_{j}\right)\right]^{2} d_{V_{j}}=1 .
$$


From the relation $\left(4^{\prime}\right)$, it may be deduced that the function $\psi^{0}(V)=\psi_{0_{x}}\left(V_{x}\right) \psi_{0_{y}}\left(V_{y}\right) \psi_{0_{z}}\left(V_{z}\right)$ is an eigenfunction of operator $A$, associated to eigenvalue $\lambda_{0_{x}} \lambda_{0_{y}} \lambda_{0_{z}}=1$ (see demonstration in appendix B). This first eigenfunction takes an important part in the solution of the problem because it corresponds to the maximal eigenvalue of $A$, and so leads to an equilibrium state. Indeed, in the equilibrium state between the gas and the wall, the most physically convenient scattering kernel is the complete accommodation kernel, which is assigned here to $\psi^{0}(V)$. Therefore any good model of a scattering kernel must converge to this equilibrium scattering kernel. This point matches the basic assumption founding the reciprocity relation which is that any scattering kernel must be valid when a thermodynamic equilibrium exists between the gas and the wall.

In agreement with the concept of three distinguishable degrees of freedom in the reflection process, we introduce three coefficients $\alpha_{j}$, related to the set of eigenvalues by: $\lambda_{0_{j}}=1$, and $\lambda_{k_{j}}=\left(1-\alpha_{j}\right)$ for $k \neq 0$ with $\alpha_{j} \in[0,1]$; relation (14) becomes

$$
K\left(V, V^{\prime}\right)=\prod_{j=1}^{3}\left(\psi_{0_{j}}\left(V_{j}\right) \psi_{0_{j}}\left(V_{j}^{\prime}\right)+\left(1-\alpha_{j}\right) \sum_{k=1}^{\infty} \psi_{k_{j}}\left(V_{j}\right) \psi_{k_{j}}\left(V_{j}^{\prime}\right)\right)
$$

which may be rewritten,

$$
K\left(V, V^{\prime}\right)=\prod_{j=1}^{3}\left(\alpha_{j} \psi_{0_{j}}\left(V_{j}\right) \psi_{0_{j}}\left(V_{j}^{\prime}\right)+\left(1-\alpha_{j}\right) \sum_{k=0}^{\infty} \psi_{k_{j}}\left(V_{j}\right) \psi_{k_{j}}\left(V_{j}^{\prime}\right)\right) .
$$

Finally, by using the following property:

$$
\sum_{k=0}^{\infty} \psi_{k_{j}}\left(V_{j}\right) \psi_{k_{j}}\left(V_{j}^{\prime}\right)=\delta\left(V_{j}-V_{j}^{\prime}\right)
$$

we obtain the result:

$$
K\left(V, V^{\prime}\right)=\prod_{j=1}^{3}\left(\alpha_{j} \psi_{0_{j}}\left(V_{j}\right) \psi_{0_{j}}\left(V_{j}^{\prime}\right)+\left(1-\alpha_{j}\right) \delta\left(V_{j}-V_{j}^{\prime}\right)\right) .
$$

By developing the product (17) we obtain $K\left(V, V^{\prime}\right)$ as a sum of elementary operators as follows:

$$
K\left(V, V^{\prime}\right)=\sum_{\kappa} \mu_{\kappa} K_{\kappa}\left(V, V^{\prime}\right)
$$


where $\mu_{\kappa}$ is given again by the relations (9) of section III, and where the elementary operators $K_{\kappa}\left(V, V^{\prime}\right)$ are written below:

$$
\begin{aligned}
& K_{0}\left(V, V^{\prime}\right)=\delta\left(V_{x}-V_{x}^{\prime}\right) \delta\left(V_{y}-V_{y}^{\prime}\right) \delta\left(V_{z}-V_{z}^{\prime}\right), \\
& K_{y z}\left(V, V^{\prime}\right)=\frac{1}{\pi C_{w}^{2}} \delta\left(V_{x}-V_{x}^{\prime}\right) e^{-\frac{V_{y}^{2}}{2 C_{w}^{2}}} e^{-\frac{V_{z}^{2}}{2 C_{w}^{2}}} e^{-\frac{V_{y}^{\prime 2}}{2 C_{w}^{2}}} e^{-\frac{V_{z}^{\prime 2}}{2 C_{w}^{2}}} \\
& K_{x z}\left(V, V^{\prime}\right)=\frac{2}{C_{w}^{3} \sqrt{\pi}}\left|V_{x} V_{x}^{\prime}\right|^{\frac{1}{2}} \delta\left(V_{y}-V_{y}^{\prime}\right) e^{-\frac{V_{x}^{2}}{2 C_{w}^{2}}} e^{-\frac{V_{z}^{2}}{2 C_{w}^{2}}} e^{-\frac{V_{x}^{\prime 2}}{2 C_{w}^{2}}} e^{-\frac{V_{z}^{\prime 2}}{2 C_{w}^{2}}} \\
& K_{x y}\left(V, V^{\prime}\right)=\frac{2}{C_{w}^{3} \sqrt{\pi}}\left|V_{x} V_{x}^{\prime}\right|^{\frac{1}{2}} \delta\left(V_{z}-V_{z}^{\prime}\right) e^{-\frac{V_{x}^{2}}{2 C_{w}^{2}}} e^{-\frac{V_{y}^{2}}{2 C_{w}^{2}}} e^{-\frac{V_{x}^{\prime 2}}{2 C_{w}^{2}}} e^{-\frac{V_{y}^{\prime 2}}{2 C_{w}^{2}}} \\
& K_{x y z}\left(V, V^{\prime}\right)=\frac{2}{\pi C_{w}^{4}}\left|V_{x} V_{x}^{\prime}\right|^{\frac{1}{2}} e^{-\frac{V_{x}^{2}}{2 C_{w}^{2}}} e^{-\frac{V_{y}^{2}}{2 C_{w}^{2}}} e^{-\frac{V_{z}^{2}}{2 C_{w}^{2}}} e^{-\frac{V_{x}^{\prime 2}}{2 C_{w}^{2}}} e^{-\frac{V_{y}^{\prime 2}}{2 C_{w}^{2}}} e^{-\frac{V_{z}^{\prime 2}}{2 C_{w}^{2}}} \\
& K_{z}\left(V, V^{\prime}\right)=\frac{1}{C_{w} \sqrt{\pi}} \delta\left(V_{x}-V_{x}^{\prime}\right) \delta\left(V_{y}-V_{y}^{\prime}\right) e^{-\frac{V_{z}^{2}}{C_{w}^{2}}} e^{-\frac{V_{z}^{\prime 2}}{C_{w}^{2}}}, \\
& K_{y}\left(V, V^{\prime}\right)=\frac{1}{C_{w} \sqrt{\pi}} \delta\left(V_{x}-V_{x}^{\prime}\right) \delta\left(V_{z}-V_{z}^{\prime}\right) e^{-\frac{V_{y}^{2}}{2 C_{w}^{2}}} e^{-\frac{V_{y}^{\prime 2}}{2 C_{w}^{2}}} \\
& K_{x}\left(V, V^{\prime}\right)=\frac{2}{C_{w}^{2}}\left|V_{x} V_{x}^{\prime}\right|^{\frac{1}{2}} \delta\left(V_{y}-V_{y}^{\prime}\right) \delta\left(V_{z}-V_{z}^{\prime}\right) e^{-\frac{V_{x}^{2}}{2 C_{w}^{2}}} e^{-\frac{V_{x}^{\prime 2}}{2 C_{w}^{2}}}
\end{aligned}
$$

It is easy to verify that the elementary operators $K_{\kappa}\left(V, V^{\prime}\right)$ given above correspond to the elementary kernels $B_{\kappa}\left(V^{\prime}, V\right)$ given in section III, through transformation (10); so the complete operator $K\left(V, V^{\prime}\right)$ obtained from relation (18) corresponds exactly to the scattering kernel given by relation (7).

Referring now directly to equations (15) and (13), it is clear that our choice corresponds to a spectral expansion of $K\left(V, V^{\prime}\right)$ involving eight different eigenvalues, namely: 1 , (1$\left.\alpha_{x}\right),\left(1-\alpha_{y}\right),\left(1-\alpha_{z}\right),\left(1-\alpha_{x}\right)\left(1-\alpha_{y}\right),\left(1-\alpha_{x}\right)\left(1-\alpha_{z}\right),\left(1-\alpha_{y}\right)\left(1-\alpha_{z}\right),\left(1-\alpha_{x}\right)(1-$ $\left.\alpha_{y}\right)\left(1-\alpha_{z}\right)$. Of course this choice also corresponds to a degeneracy of the eigenfunction 
space associated to each eigenvalue, except for the maximum eigenvalue; therefore, as shown above, it is not necessary to specify the eigenfunctions.

As is well known, the classical CL model and its extensions by Lord [13, 14] to polyatomic molecules are derived from another procedure based on a more general relation [12]:

$$
K\left(V, V^{\prime}\right)=\sum_{n, m=0}^{\infty} \lambda^{n m} \varphi^{n}(V) \varphi^{m}\left(V^{\prime}\right)
$$

where the $\varphi^{k}$ function is specified. As it can be seen, kernels built on this general form could not automatically insure the properties listed above for good scattering kernels (i.e positivity, normalization, and reciprocity properties)[12]. Moreover, in these CL scattering kernel models the normal and the tangential components of the velocity of the molecule colliding with the wall are considered as independent, and then the scattering kernel is written separately in normal and tangential parts. This aspect of the CL models was recently criticized as not allowing interplay between the various components of the impinging particle velocity [5].

\section{RELATIONS WITH ACCOMMODATION COEFFICIENTS}

In this section the physical meaning of the parameters involved in the kernel is clarified. So doing, we prove that the $\alpha_{j}$ coefficients respectively equal the accommodation coefficients $\beta_{j}$ of the momentum components.

First $\mu_{\kappa}$ may be easily shown to represent the weight of particles reflected according to the process $\kappa$ in the flux of reflected particles. This property appears obvious when integrating the two members of equation (3) over the reflected velocity range, using $B\left(V^{\prime}, V\right)$ expressions given in relations (7) and (9). Moreover the scattering is built using three independent coefficients $\alpha_{j}$ : then it can be seen that taking into account condition (8), the $\mu_{\kappa}$ coefficients are necessarily given by relations (9).

On the other hand, the momentum accommodation coefficients $\beta_{j}$ are defined as [9]:

$$
\beta_{j}=\frac{\Phi_{j}^{-}-\Phi_{j}^{+}}{\Phi_{j}^{-}-\Phi_{j}^{e}} .
$$


where $\Phi_{j}^{-}$is the incoming flux at the wall of the momentum $j$ component, $\Phi_{j}^{+}$is the the corresponding reflected flux, and $\Phi_{j}^{e}$ is the reflected flux in the hypothetical situation of perfect accommodation to the wall. These various momentum fluxes may be written for each component:

$$
\begin{gathered}
\Phi_{j}^{-}=\int_{\Omega^{\prime}} m\left|V_{x}^{\prime}\right| V_{j}^{\prime} f^{-}\left(V^{\prime}\right) d_{V^{\prime}} \\
\Phi_{j}^{+}=\int_{\Omega} m\left|V_{x}\right| V_{j} f^{+}(V) d_{V}
\end{gathered}
$$

where $f^{-}(V)$ and $f^{+}(V)$ are respectively the incident and the reflected distribution function. Remembering that $f^{+}(V)$ is given by (3) and using expression (7) of $B\left(V^{\prime}, V\right), \Phi_{j}^{+}$is written:

$$
\Phi_{j}^{+}=\int_{\Omega^{\prime}} m\left|V_{x}^{\prime}\right| f^{-}\left(V^{\prime}\right) \sum_{\kappa} \mu_{\kappa}\left(\int_{\Omega} V_{j} B_{\kappa}\left(V^{\prime}, V\right) d_{V}\right) d_{V^{\prime}} .
$$

And $\Phi_{j}^{e}$ for total perfect accommodation (i.e. $\alpha_{x}=\alpha_{x}=\alpha_{x}=1$ ) is written:

$$
\Phi_{j}^{e}=\int_{\Omega^{\prime}} m\left|V_{x}^{\prime}\right| f^{-}\left(V^{\prime}\right)\left(\int_{\Omega} V_{j} B_{x y z}\left(V^{\prime}, V\right) d_{V}\right) d_{V^{\prime}}
$$

\section{A. calculation of $\beta_{y}$ and $\beta_{z}$}

First the case $V_{j}=V_{y}$ is dealt with.

According to the expression of $B_{x y z}\left(V^{\prime}, V\right), V_{y} B_{x y z}\left(V^{\prime}, V\right)$ is an odd function of the $V_{y}$ component, therefore,

$$
\int_{\Omega} V_{y} B_{x y z}\left(V^{\prime}, V\right) d_{V}=0
$$

and then $\Phi^{e}=0$, so we can write

$$
\beta_{y}=1-\frac{\Phi_{y}^{+}}{\Phi_{y}^{-}}
$$

On the other hand looking at relation (23), the contribution of each partial kernel $B_{\kappa}\left(V^{\prime}, V\right)$ in $\Phi_{y}^{+}$expression can be estimated separately by:

$$
\int_{\Omega} V_{y} B_{\kappa}\left(V^{\prime}, V\right) d_{V}
$$


Each of these partial integrals can be calculated easily and give for any $\kappa$ either 0 or $V_{y}^{\prime}$. As result we obtain

$$
\sum_{\kappa} \mu_{\kappa} \int_{\Omega} V_{y} B_{\kappa}\left(V^{\prime}, V\right) d_{V}=V_{y}^{\prime}\left(\mu_{0}+\mu_{x}+\mu_{z}+\mu_{x z}\right)
$$

and

$$
\Phi_{j}^{+}=\left(\mu_{0}+\mu_{x}+\mu_{z}+\mu_{x z}\right) \Phi_{j}^{-}
$$

It results directly that

$$
\beta_{y}=1-\left(\mu_{0}+\mu_{x}+\mu_{z}+\mu_{x z}\right)
$$

which, according to the normalization condition (8), is also equal to

$$
\beta_{y}=\mu_{y}+\mu_{x y}+\mu_{y z}+\mu_{x y z}
$$

Similarly we obtain for $\beta_{z}$

$$
\beta_{z}=\mu_{z}+\mu_{x z}+\mu_{y z}+\mu_{x y z}
$$

By using the $\mu_{\kappa}$ expressions given by relations (9), equations (25) and (26) lead to:

$$
\beta_{y}=\alpha_{y}
$$

and

$$
\beta_{z}=\alpha_{z}
$$

\section{B. calculation of $\beta_{x}$}

Now $V_{j}=V_{x}$ is considered.

Using the same definition of the various fluxes with respect to the sign of the incident flux, we can write:

$$
\Phi_{x}^{-}=\int_{\Omega^{\prime}} m V_{x}^{\prime 2} f^{-}\left(V^{\prime}\right) d_{V^{\prime}}
$$

The calculation of the partial integrals involved in relation (23)

$$
\int_{\Omega} V_{x} B_{\kappa}\left(V^{\prime}, V\right) d_{V}
$$


gives in this case, either $-V_{x}^{\prime}$ or $\frac{C_{w} \sqrt{\pi}}{2}$ for any $\kappa$. As a result we obtain:

$$
\begin{array}{r}
\sum_{\kappa} \mu_{\kappa} \int_{\Omega} V_{x} B_{\kappa}\left(V^{\prime}, V\right) d_{V}=-V_{x}^{\prime}\left(\mu_{0}+\mu_{y}+\mu_{z}+\mu_{y z}\right) \\
+\frac{C_{w} \sqrt{\pi}}{2}\left(\mu x+\mu_{x y}+\mu_{x z}+\mu_{x y z}\right) .
\end{array}
$$

Using the flux expressions (29) and (23), this result leads to:

$$
\begin{array}{r}
\Phi_{x}^{-}-\Phi_{x}^{+}=\left(1-\left(\mu_{0}+\mu_{y}+\mu_{z}+\mu_{y z}\right)\right) \Phi_{x}^{-}- \\
\frac{C_{w} \sqrt{\pi}}{2}\left(\mu_{x}+\mu_{x y}+\mu_{x z}+\mu_{x y z}\right) \int_{\Omega^{\prime}} m\left|V_{x}^{\prime}\right| f^{-}\left(V^{\prime}\right) d_{V^{\prime}}
\end{array}
$$

We also have:

$$
\int_{\Omega} V_{x} B_{x y z}\left(V^{\prime}, V\right) d_{V}=\frac{C_{w} \sqrt{\pi}}{2}
$$

and so:

$$
\Phi_{x}^{-}-\Phi_{x}^{e}=\Phi_{x}^{-}-\frac{C_{w} \sqrt{\pi}}{2} \int_{\Omega^{\prime}} m\left|V_{x}^{\prime}\right| f^{-}\left(V^{\prime}\right) d_{V}^{\prime} .
$$

Consequently, remembering condition (8), it results

$$
\beta_{x}=\mu_{x}+\mu_{x y}+\mu_{x z}+\mu_{x y z}
$$

Replacing $\mu_{\kappa}$ by relations (9) once more, the result shows:

$$
\beta_{x}=\alpha_{x}
$$

The classical Maxwell model predicted the same value of the various accommodation coefficients [9]. However it is well-known, notably from the measurements of accommodation coefficients by various procedures [15-17], that these coefficients do not have the same value; and this description of equivalent accommodation is not physically consistent [18]. In the new model we can see that the three parameters introduced in the theoretical modelling equal the three accommodation coefficients of momentum component fluxes which are basically different. 


\section{EXTENSION OF THE NEW MODEL TO POLYATOMIC GASES}

In polyatomic gases the internal state of a molecule is characterized by the rotational and vibrational energies, $E_{i r}$ and $E_{i v}$, and depends on quantum numbers $i r$ and $i v$. In addition, because of the degeneracy of rotational levels, a weight factor $\left(g_{i r}=2 i r+1\right)$ is involved in the distribution function expression. The distribution functions of impinging and emerging particles are linked by the following relation (generalizing relation $(3)$ ) $[9,19]$ :

$$
\begin{array}{r}
V_{x} f_{i}^{+}\left(V, E_{i r}, g_{i r}, E_{i v}\right)= \\
\sum_{i r^{\prime}, i v^{\prime}} \int_{\Omega^{\prime}}\left|V_{x}^{\prime}\right| f^{-}\left(V^{\prime}, E_{i r^{\prime}}, g_{i r^{\prime}}, E_{i v^{\prime}}\right) P_{G}\left(V^{\prime}, E_{i r^{\prime}}, g_{i r^{\prime}}, E_{i v^{\prime}}, V, E_{i r}, g_{i r}, E_{i v}\right) d_{V^{\prime}}
\end{array}
$$

where $P_{G}\left(V^{\prime}, E_{i r^{\prime}}, g_{i r^{\prime}}, E_{i v^{\prime}}, V, E_{i r}, g_{i r}, E_{i v}\right)$ is the generalized scattering kernel for molecules considered with internal modes. Of course normalization and non-negative conditions are easily generalized to:

$$
\sum_{i r, i v} \int_{\Omega} P_{G}\left(V^{\prime}, E_{i r^{\prime}}, g_{i r^{\prime}}, E_{i v^{\prime}}, V, E_{i r}, g_{i r}, E_{i v}\right) d_{V}=1
$$

and

$$
P_{G}\left(V^{\prime}, E_{i r^{\prime}}, g_{i r^{\prime}}, E_{i v^{\prime}}, V, E_{i r}, g_{i r}, E_{i v}\right) \geq 0
$$

Furthermore, for the reciprocity condition, we admit the form given by Kuscer [9] which, excluding external magnetic fields, assumes the form:

$$
\begin{gathered}
\left|V_{x}^{\prime}\right| e^{-\frac{\left\|V^{\prime}\right\|^{2}}{C_{w}^{2}}} e^{-\varepsilon_{i r^{\prime}}} e^{-\varepsilon_{i v^{\prime}}}\left(2 i r^{\prime}+1\right) P_{G}\left(V^{\prime}, E_{i r^{\prime}}, E_{i v^{\prime}}, V, E_{i r}, E_{i v}\right)= \\
V_{x} e^{-\frac{\|V\|^{2}}{C_{w}^{2}}} e^{-\varepsilon_{i r}} e^{-\varepsilon_{i v}}(2 i r+1) P_{G}\left(-V, E_{i r}, E_{i v},-V^{\prime}, E_{i r^{\prime}}, E_{i v^{\prime}}\right)
\end{gathered}
$$

with

$$
\varepsilon_{i r}=\frac{E_{i r}}{k T_{w}}, \quad \varepsilon_{i v}=\frac{E_{i v}}{k T_{w}} .
$$

Reference [19] presents, phenomenologically, a scattering kernel $P$ characterizing the interaction of diatomic molecules with internal modes at the wall (this kernel $P$ is recalled in 
the appendix $\mathrm{C}$ ). In kernel $P$ the translation mode was taken as a whole (without interplay between the three translational degrees of freedom); so, when applied to unstructured molecules, $P$ reduced to a classical Maxwell kernel. Nevertheless, a partial accommodation concept was employed in the $P$ derivation allowing different accommodations of the various modes (translation, rotation, vibration). In fact this concept was analogous to those used in the new model to disconnect the three translation degrees in the accommodation process. From reference [19], $P$ may be immediately rewritten:

$$
\begin{array}{r}
P=\left(\left(1-\alpha_{\theta}\right) \delta\left(V-V_{R}^{\prime}\right)+\alpha_{\theta} \frac{2}{C_{w}^{4} \pi} V_{x} e^{-\frac{V^{2}}{C_{w}^{2}}}\right) \\
\left(\left(1-\alpha_{r}\right)\left(1-\alpha_{v}\right) \tilde{P}_{0}+\alpha_{v}\left(1-\alpha_{r}\right) \tilde{P}_{v}+\alpha_{r}\left(1-\alpha_{v}\right) \tilde{P}_{r}+\alpha_{v} \alpha_{r} \tilde{P}_{r v}\right)
\end{array}
$$

where $\alpha_{\theta}, \alpha_{v}$ and $\alpha_{r}$ are real parameters independent of the microscopic state of the molecules and are considered in $[0,1]$, and :

$$
\begin{aligned}
& \tilde{P}_{0}=\delta\left(E_{i r^{\prime}}-E_{i r}\right) \delta\left(E_{i v^{\prime}}-E_{i v}\right), \quad \tilde{P}_{r v}=\frac{e^{-\varepsilon_{i r}}}{Q_{r}}\left(2 i_{r}+1\right) \frac{e^{-\varepsilon_{i v}}}{Q_{v}}, \\
& \tilde{P}_{v}=\delta\left(E_{i r^{\prime}}-E_{i r}\right) \frac{e^{-\varepsilon_{i v}}}{Q_{v}}, \quad \tilde{P}_{r}=\frac{e^{-\varepsilon_{i r}}}{Q_{r}}(2 i r+1) \delta\left(E_{i v^{\prime}}-E_{i v}\right) .
\end{aligned}
$$

The quantities $Q_{v}$ and $Q_{r}$ involved in $\tilde{P}_{\chi}(\chi=v, r, r v)$ are the partition functions defined at the wall temperature:

$$
Q_{r}=\sum_{i v}(2 i r+1) e^{-\varepsilon_{i r}}, \quad Q_{v}=\sum_{i v} e^{-\varepsilon_{i v}}
$$

Now, in operator $P(36)$, let us replace the first parenthesis corresponding to the classical Maxwell scattering kernel by the new kernel proposed in relation (7) and (9). So a general scattering kernel is obtain in the form:

$$
\begin{array}{r}
P_{G}=\left(\sum_{\kappa} \mu_{\kappa}^{*} B_{\kappa}\left(V^{\prime}, V\right)\right)\left(\left(1-\alpha_{r}\right)\left(1-\alpha_{v}\right) \tilde{P}_{0}+\right. \\
\left.\alpha_{v}\left(1-\alpha_{r}\right) \tilde{P}_{v}+\alpha_{r}\left(1-\alpha_{v}\right) \tilde{P}_{r}+\alpha_{v} \alpha_{r} \tilde{P}_{r v}\right)
\end{array}
$$

where the $\mu_{\kappa}^{*}$ are expressed by relations (9) using the directional parameters $\alpha_{x}^{*}, \alpha_{y}^{*}$, and $\alpha_{z}^{*}$. The subscript $\left(^{*}\right)$ is used to indicate that the physical meaning of the three parameters is not yet shown in the polyatomic modelling frame. 
Considering the properties of its parts, $P_{G}$ visibly satisfies the normalization and nonnegativity conditions. Furthermore $P_{G}$ is a sum of partial operators assuming the factorized form $\tilde{P}_{\chi} B_{\kappa}$ (where $\chi$ refers to the internal accommodation process). It is clear that the $B_{\kappa}$ satisfying condition (4) implies that $\tilde{P}_{\chi} B_{\kappa}$ satisfies the generalized reciprocity condition (34). Consequently $P_{G}$ also satisfies (34) as a linear combination of $\tilde{P}_{j} B_{\kappa}$ terms. So a good anisotropic scattering kernel $P_{G}$ has been obtained phenomenologically.

Furthermore in relation (37) the part in the first parenthesis of the second member concerns the translation mode. Introducing into it $\alpha_{\theta}$ defined as $\alpha_{\theta}=1-\mu_{0}^{*}=\sum_{\kappa \neq 0} \mu_{\kappa}^{*}$, this part assumes the form:

$$
P_{G}=\left(1-\alpha_{\theta}\right) \delta\left(V-V_{R}^{\prime}\right)+\alpha_{\theta} \sum_{\kappa \neq 0} \lambda_{\kappa}^{*} B_{\kappa}\left(V^{\prime}, V\right)
$$

where

$$
\lambda_{\kappa}^{*}=\frac{\mu_{\kappa}^{*}}{\alpha_{\theta}}, \quad \sum_{\kappa \neq 0} \lambda_{\kappa}^{*}=1 .
$$

In this last form the complete specular reflection appears separately from all the processes in which partial or complete accommodation occurs.

Integrating on velocity and summing over quantum numbers the left and right sides of equation (31), it is easily shown that $\mu_{\kappa}^{*}$ represents (as $\mu_{\kappa}$ for the unstructured molecules) the part of the particle flux reflected according to the directional process $\kappa$. The analytical systematic derivation of $P_{G}$ and further physical interpretation of $\alpha_{x}^{*}, \alpha_{y}^{*}, \alpha_{z}^{*}, \alpha_{v}, \alpha_{r}$, and $\alpha_{\theta}$ will be presented in a subsequent study.

Regarding now the process from the point of view of energy transfer, the comments given in reference [19] can be extended. The thermal kinetic energy of the particle flow may be exchanged in collisions at the wall: at the statistical level the specular kernels reflect the effect of elastic collisions at the wall for the translational mode, while diffusive kernels represent the effects of inelastic collisions for this same mode. In the present model the influence of these inelastic collisions is considered independently for each translational degree (i.e. for each direction). Statistically the scattered distribution function is no longer 
accommodated in the same way according to the normal and tangential directions: and on the microscopic level this means that the kinetic energy transfers of an impinging molecule change according to its velocity direction with respect to the wall.

In the same manner the three energy modes may interplay when exchanging energy at the wall; and so in operators $\tilde{P}_{0}, \tilde{P}_{r}, \tilde{P}_{v}$, and $\tilde{P}_{r v}$ the effects of elastic or inelastic collisions for each internal mode appear independently.

\section{DISCUSSION}

The new model proposed in this paper presents many aspects which allow realistic gas surface interactions to be described. First, the model takes into account the anisotropic effects in the interacting process at the wall. This anisotropic character of the surface derives basically from its physical properties and/or from its suitable treatment. Consequently the real physical properties of the wall are embedded in the three accommodation coefficients of the momentum components. Existing models rarely involve three directional parameters as the present model does: the former approaches using a three parameters representation $[2$, 20] are based on the suggestion of a shifted Maxwellian to describe the re-emitted distribution function of a monatomic gas from a solid surface and the parameters are arbitrary constants [21]. Here the new model gives an anisotropic description of the interaction in the sense employed by Kuscer [9] i.e. the new kernel operator is no longer invariant under rotation about the normal axis at the solid surface; moreover in the new model the parameters are shown to be the accommodation coefficients themselves so it is possible to relate them to physical measurements $[15-18,22]$. In addition, in polyatomic cases, the present model allows independent accommodation processes at the wall for the various energy modes which seems a realistic description if, for example, the very different situations occurring at the wall for vibrational or translational accommodation are kept in mind [23, 24]. Then, it is to be noted that the present model also appears as an improvement with respect to two well-known criticisms opposed to the methods based on the scattering kernel concept. The 
first criticism formulated by Cercignani [3] concerns the Maxwellian-type kernel: for a given monochromatic beam this model predicts a sharp maximum in the number of molecules at the angle corresponding to specular reflection, which is contradicted by experiments [25]; it is clear that this maximum is smoothed in the new model (see relation (38)). The second criticism recently formulated by D. Bruno et al [5], is not completely justified because it is based, for a part, on studies of reactive or dissociated flows; and as is well-known, the scattering kernel modelling cannot give correct results in such a case because of the assumption of the wall impermeability involved in it. But on the other hand, the comments of the authors requiring the introduction of interplay between the velocity directions and the various energy modes is, in our opinion, completely justified. In the present model, precisely such an interplay is present, and the separating procedure of velocity components, which appears in the CL models [3](also when extended to internal modes $[13,14,26]$ ) is not employed here.

A final argument in defence of the new model is its simplicity: as seen above the physical interpretation of the various parts of the kernel is straightforward. Moreover the model is easy-to-use: first the calculations are simplified because the kernel only depends on the reflected microscopic parameters space; then, from the account of different kinds of accommodation of the moment components a preponderant weight can be given to the particular accommodation process; so the model may be easily simplified according to the geometrical symmetry or the physical conditions of the problem.

\section{CONCLUDING REMARKS}

We have derived an anisotropic model of a scattering kernel. For the unstructured molecules, three directional parameters involved in the model have been shown to be equal to the accommodation coefficients of the fluxes of the momentum components at the wall.

We have also extended the new model to polyatomic structured molecules. In this domain only a phenomenological derivation has been presented. The corresponding analytical 
method and further calculations to relate parameters $\alpha_{x}^{*}, \alpha_{y}^{*}, \alpha_{z}^{*}, \alpha_{v}, \alpha_{r}, \alpha_{\theta}$ to the accommodation coefficients $\beta_{j}$ of momentum fluxes and to accommodation coefficients of the energy fluxes will be presented in a subsequent study. These relationships are not expected to be very simple, especially because the constant parameters introduced in the model refer to direct exchanges between each molecular mode and the wall, while the energy accommodation coefficients involve direct exchange with the wall for each mode and also inter-mode exchanges. Finally, even if not yet confirmed in applications, many arguments have been developed, showing the consistency, physical pertinence and usefulness of the new scattering kernel.

\section{APPENDIX A:}

\section{$B_{\kappa}\left(V^{\prime}, V\right)$ satisfies the reciprocity relation:}

The property for operator $B_{z y}$ is demonstrated. For the rest of the operators the demonstrations are similar.

$$
\left|V_{x}^{\prime}\right| f_{0}\left(V^{\prime}\right) B_{z y}\left(V^{\prime}, V\right)=\left|V_{x}^{\prime}\right| \frac{n}{\left(C_{w} \sqrt{\pi}\right)^{3}} e^{-\frac{\left\|V^{\prime}\right\|^{2}}{C_{w}^{2}}} \frac{1}{\pi C_{w}^{2}} \delta\left(V_{x}+V_{x}^{\prime}\right) e^{-\frac{V_{y}^{2}}{C_{w}^{2}}} e^{-\frac{V_{z}^{2}}{C_{w}^{2}}}
$$

and

$$
\left|V_{x}\right| f_{0}(-V) B_{z y}\left(-V,-V^{\prime}\right)=\left|V_{x}\right| \frac{n}{\left(C_{w} \sqrt{\pi}\right)^{3}} e^{-\frac{\|V\|^{2}}{C_{w}^{2}}} \frac{1}{\pi C_{w}^{2}} \delta\left(V_{x}+V_{x}^{\prime}\right) e^{-\frac{V_{y}^{\prime 2}}{C_{w}^{2}}} e^{-\frac{V_{z}^{\prime 2}}{C_{w}^{2}}} .
$$

In these two expressions, there appear the same terms in $y$ and $z$; the full equality of both the expressions comes from the property of the dirac function.

\section{APPENDIX B:}

$\psi^{0}$ as an eigenfunction of operator $A$ :

$$
A\left(\psi^{0}\right)=\int_{\Omega^{\prime}}\left[\left|V_{x}^{\prime}\right| f_{0}\left(V^{\prime}\right)\right]^{\frac{1}{2}}\left[\left|V_{x}\right| f_{0}(V)\right]^{\frac{-1}{2}} B\left(V_{R}^{\prime}, V\right) \psi^{0}\left(V^{\prime}\right) d_{V^{\prime}}
$$


Replacing $\psi^{0}$ and $f_{0}(V)$ by their expression in this integral: $\psi^{0}=\frac{\sqrt{2}}{C_{w}^{2} \sqrt{\pi}}\left|V_{x}\right|^{\frac{1}{2}} e^{-\frac{\|V\|^{2}}{2 C_{w}^{2}}}$ and $f_{0}(V)=\frac{n}{\left(C_{w} \sqrt{\pi}\right)^{3}} e^{-\frac{\|V\|^{2}}{C_{w}^{2}}}$, it is found

$$
A\left(\psi^{0}\right)=\frac{\sqrt{2}}{C_{w}^{2} \sqrt{\pi}} \int_{\Omega^{\prime}}\left|V_{x}\right|^{\frac{-1}{2}}\left|V_{x}^{\prime}\right| e^{\frac{\|V\|^{2}}{2 C_{w}^{2}}} e^{-\frac{\left\|V^{\prime}\right\|^{2}}{C_{w}^{2}}} B\left(V_{R}^{\prime}, V\right) d_{V^{\prime}} .
$$

The reciprocity relation $\left(4^{\prime}\right)$ leads to:

$$
\int_{\Omega^{\prime}}\left|V_{x}^{\prime}\right| e^{-\frac{\left\|V^{\prime}\right\|^{2}}{C_{w}^{2}}} B\left(V_{R}^{\prime}, V\right) d_{V^{\prime}}=\left|V_{x}\right| e^{-\frac{\|V\|^{2}}{C_{w}^{2}}} .
$$

And consequently

$$
A\left(\psi^{0}\right)=\frac{\sqrt{2}}{C_{w}^{2} \sqrt{\pi}}\left|V_{x}\right|^{\frac{1}{2}} e^{-\frac{\|V\|^{2}}{2 C_{w}^{2}}}=\psi^{0} .
$$

\section{APPENDIX C:}

\section{Kernel Operator of reference [19]}

The full scattering kernel of reference [19] is given by:

$$
\begin{array}{r}
P=\left(1-\alpha_{\theta}\right)\left(1-\alpha_{r}\right)\left(1-\alpha_{v}\right) P_{1}+\left(1-\alpha_{\theta}\right)\left(1-\alpha_{r}\right) \alpha_{v} P_{2}+\left(1-\alpha_{\theta}\right) \alpha_{r}\left(1-\alpha_{v}\right) P_{3} \\
+\left(1-\alpha_{\theta}\right) \alpha_{r} \alpha_{v} P_{4}+\alpha_{\theta}\left(1-\alpha_{r}\right)\left(1-\alpha_{v}\right) P_{5}+\alpha_{\theta} \alpha_{r}\left(1-\alpha_{v}\right) P_{6}+\alpha_{\theta}\left(1-\alpha_{r}\right) \alpha_{v} P_{7}+\alpha_{\theta} \alpha_{r} \alpha_{v} P_{8}
\end{array}
$$

where $\alpha_{\theta}, \alpha_{r}, \alpha_{v}$ are real parameters independent of the microscopic state of the molecules. The specular operator with elastic and inelastic parts for each internal mode are given by:

$$
\begin{gathered}
P_{1}=\delta\left(V^{\prime}-V_{R}\right) \delta\left(E_{i r}^{\prime}-E_{r}\right) \delta\left(E_{i v}^{\prime}-E_{v}\right), \\
P_{2}=\delta\left(V^{\prime}-V_{R}\right) \delta\left(E_{i r}^{\prime}-E_{r}\right) \frac{e^{-\varepsilon_{i v, w}}}{Q_{v, w}}, \\
P_{3}=\delta\left(V^{\prime}-V_{R}\right) \frac{e^{-\varepsilon_{i r, w}}}{Q_{r, w}}(2 i r+1) \delta\left(E_{i v}^{\prime}-E_{v}\right), \\
P_{4}=\delta\left(V^{\prime}-V_{R}\right) \frac{e^{-\varepsilon_{i r, w}}}{Q_{r, w}}(2 i r+1) \frac{e^{-\varepsilon_{i v, w}}}{Q_{v, w}} .
\end{gathered}
$$


The diffusive operators with elastic and inelastic parts, for each internal mode, are given by:

$$
\begin{gathered}
P_{5}=\frac{2}{\pi C_{w}^{4}} V_{x} e^{-v_{w}^{2}} \delta\left(E_{i r}-E_{i r}^{\prime}\right) \delta\left(E_{i v}-E_{i v}^{\prime}\right), \\
P_{6}=\frac{2}{\pi C_{w}^{4}} V_{x} e^{-v_{w}^{2}} \frac{e^{-\varepsilon_{i r, w}}}{Q_{r, w}}(2 i r+1) \delta\left(E_{i v}-E_{i v}^{\prime}\right), \\
P_{7}=\frac{2}{\pi C_{w}^{4}} V_{x} e^{-v_{w}^{2}} \delta\left(E_{i r}-E_{i r}^{\prime}\right) \frac{e^{-\varepsilon_{i v, w}}}{Q_{v, w}} \\
P_{8}=\frac{2}{\pi C_{w}^{4}} V_{x} e^{-v_{w}^{2}} \frac{e^{-\varepsilon_{i r, w}}}{Q_{r, w}}(2 i r+1) \frac{e^{-\varepsilon_{i v, w}}}{Q_{v, w}}
\end{gathered}
$$

with

$$
\frac{1}{C_{w}^{4}}=\left(\frac{m}{2 k T_{w}}\right)^{2} .
$$

In reference [19], $E_{r}$ and $E_{v}$ defined as mean energy per molecule have been used by error. The correct parameters to use anywhere in this reference are $E_{i r}$ and $E_{i v}$. 
[1] J. C. Maxwell, Philosophical Transactions of the royal society of London 170, 231 (1878).

[2] S. F. Shen, Entropie 18, 138 (1967).

[3] C. Cercignani and M. Lampis, transport theory and statistical physics 1, 101 (1971).

[4] C. Cercignani and M. Lampis, AIAA journal 35 (1997).

[5] D.Bruno, M. Cacciatore, S. Longo, and M. Rutigliano, chemical physics letters 320, 245 (2000).

[6] R. Monaco, Introduction à la théorie et aux applications de l'interaction gaz-paroi en théorie cinétique des gaz, Dipartimento di Matematica Politecnico Torino, Torino-Corso, libreria editrice universitaria levrotto \& bella ed. (1986).

[7] C. Cercignani, Theory and Application of the Boltzmann Equation (Scottish Academic Press, Edinburgh, 1975).

[8] S. Chapman and T. Cowling, The Mathematical Theory of Non-Uniform Gases (Cambridge Mathematical Library, 1970), third edition ed.

[9] I. Kuscer, in International Symposium on Rarefied Gas Dynamics, Gottingen (1974), vol. E1, pp. $1-21$.

[10] F. Sharipov, European Journal of Mechanics, B/Fliuds 21 (2001).

[11] C. Cercignani, Journal of Statistical Physics 1, 297 (1969).

[12] C. Cercignani, Mathematical Methods in Kinetic Theory (plenum press new york, 1990), chap. 4, p. 98, 2nd ed.

[13] R. G. Lord, Physic of Fluids A3, 706 (1991).

[14] R. G. Lord, Physic of Fluids 7, 1159 (1995).

[15] R. K. Hason, Physics of Fluids 16 (1973).

[16] I. Larina and V. Rykov, in $15^{\text {th }}$ Rarefied Gas Dynamics International Symposium, Teubner (1986), p. 635 .

[17] M. Seidl and E. Steinheil, Rarefied Gas Dynamics 9, E9.1 (1974). 
[18] F. Goodman, Physical Review A 68 (2003).

[19] J. G. Méolans and L. Aufrère, C. R. Acad. Sci. Paris, Mécanique des fluides/fluids mechanics t.329 (2001).

[20] C. Cercignani and M. Lampis, Mechanics Research Communication 26, 451 (1999).

[21] S. Nocilla, in $3^{\text {th }}$ Rarefied Gas Dynamics International Symposium, Aachen, edited by J. Laurman (1963).

[22] E. B. Arkilic, K. S.Breuer, and M. A. Schmidt, Journal of Fluids Mechanics 437, 29 (2001).

[23] J. G. Méolans and I. A. Graur, in $23^{\text {rd }}$ International Symposium on Rarefied Gas Dynamics, Whistler, British Columbia, Canada (2002).

[24] J. G. Méolans, accepted in press, Mechanics Research Communications, Elsevier MRC 813, editorial reference: 02-40 (2003).

[25] J. J. Hinchen and W. M. Foley, in Proceding Rarefied Gas Dynamics, edited by J. H.Leauw (Press Inc. new york, 1966).

[26] R. G. Lord, in $17^{\text {th }}$ Rarefied Gas Dynamics International Symposium, Aachen, edited by E. Alfred (Beylich, 1990). 


\section{FIGURES}




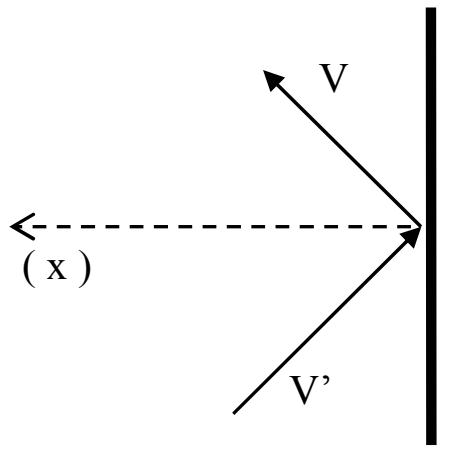

FIG. 1: particle hitting the wall. 